\title{
An Electronic Version of Volume I of the General Catalogue of Variable Stars with Improved Coordinates
}

\author{
N. N. Samus'1,2, *, V. P. Goranskii ${ }^{2}$, O. V. Durlevich ${ }^{2}$, A. V. Zharova ${ }^{2}$, \\ E. V. Kazarovets' ${ }^{1}$, E. N. Pastukhova ${ }^{1}$, M. L. Hazen ${ }^{3}$, and T. M. Tsvetkova ${ }^{1}$ \\ ${ }^{1}$ Institute of Astronomy, Russian Academy of Sciences, \\ ul. Pyatnitskaya 48, Moscow, 109017 Russia \\ ${ }^{2}$ Sternberg Astronomical Institute, Universitetskii pr. 13, Moscow, 119899 Russia \\ ${ }^{3}$ Harvard Observatory, 60, Garden Str., Cambridge, MA, 02138 USA
}

Received October 17, 2001

\begin{abstract}
We present a new electronic version of the first volume of the General Catalogue of Variable Stars (GCVS), fourth edition, which contains data on 10558 variable stars in Andromeda-Crux (the constellations are ordered in the Catalog according to the Latin alphabet). This version incorporates the name lists of variable stars from no. 67 to no. 76 for the same constellations. The main distinctive feature of the new version is that it provides improved equatorial J2000.0 coordinates for 10349 stars (including those for 4587 stars corrected for proper motions). These are based on identifications with positional catalogs using finding charts and on our new measurements. We searched for a number of stars on original plates from the plate collections of several observatories. The version also includes a file of remarks to the first GCVS volume and a preliminary version of the file of bibliographic references to the entire fourth edition of the GCVS. Apart from a complete update of the positional information, the new version incorporates all the other corrections that were found to be necessary after the first GCVS volume was published (1985).

(c) 2002 MAIK “Nauka/Interperiodica”.
\end{abstract}

Key words: stars-variable and peculiar

\section{INTRODUCTION}

The fourth edition of the General Catalogue of Variable Stars (GCVS; Kholopov et al. 1985-1988) contains data on 28435 Galactic variables in its volumes I-III (not counting the named variables that proved to be nonexistent: minor planets mistaken for stars, artifacts due to double plate exposures, etc.). Given the ten subsequent name lists of variable stars (from no. 67 to no. 76 ), the number of named Galactic variable stars in 2001 exceeded 37300.

Traditionally, the GCVS presented the coordinates of variable stars to within $1 \mathrm{~s}$ in right ascension and 0.1 arcmin in declination. In general, this accuracy corresponded to the accuracy of positional information in the original publications on the discovery of each variable star; in comparatively rare cases, the originally more accurate coordinates in the GCVS were rounded off. An appreciable fraction of the GCVS stars have even rougher coordinates than the standard accuracy or, occasionally, simply erroneous coordinates. Such a low positional accuracy makes it impossible to identify faint variable

${ }^{*}$ E-mail: samus@sai.msu.ru stars from their coordinates in the absence of finding charts. The automatic pointing of ground-based and space-borne telescopes at these objects is not possible either. In recent years, numerous projects aimed, among other tasks, at the mass automatic discovery of variable stars in fairly extended areas (MACHO, OGLE, ASAS, and others) have been under way. The impossibility of quick automatic check of the lists of new discoveries for the presence of objects identical to the already known variables, because the GCVS coordinates are too rough, prevents these stars from being included in the GCVS system.

Thus, it seems of current interest to prepare a GCVS version with improved coordinates for all the cataloged stars, wherever possible. If a star's proper motion could be found in existing positional catalogs, then we decided to include it and to take it into account in our version.

Here, we present a new version of the catalog for Volume I of the GCVS. Subsequently, we are planning to publish new versions of the GCVS Volumes II and III, as well as of the catalog of suspected variable stars (the NSV catalog). We are currently actively working on these new versions. 


\section{METHODS FOR PREPARING THE CATALOG}

With the appearance of new extensive positional catalogs, primarily the A1.0/A2.0 catalogs of the US Naval Observatory (USNO A1.0/A2.0; Monet et al. 1998) containing about 500000000 stars, the problem formulated above can now be effectively solved. For stars as faint as about $21^{m}$, these catalogs provide the coordinates determined from Schmidt-telescope survey plates that are accurate enough for our purposes, as well as their blue and red magnitudes.

The existence of Digitized Sky Survey (DSS ) images for any region of the sky also opens up great possibilities. Owing to the variable-star database maintained by the GCVS team, we have the most complete information on published finding charts of variable stars at our disposal; unpublished charts for several thousand stars were provided by their discoverers and other researchers. Finally, we have the Sternberg Astronomical Institute (SAI) plate collection at our disposal, which allows the variable stars "lost" because of the lack of finding charts and inaccurate coordinates to be rediscovered in many regions of the sky. In our work, we also used the plate collections of other observatories. The world's richest Harvard Observatory plate stacks (USA) contain the numerous marks left by the discoverers directly on plates, which allows variable stars to be found (among some 13000 variables discovered at the Harvard Observatory, about 3000 objects have no published finding charts). For several stars without charts, a clear variability can be revealed by a comparison of catalogs or DSS images. The possibility of judging the colors of stars offered by the USNO catalogs is very useful in identifying strongly colored objects (e.g., Mira-type variables). Unfortunately, the red and blue magnitudes for many fields refer to the plate epochs separated in time by a long interval, and the color indices of highly variable stars can be fictitious, reflecting primarily the variability between the two epochs rather than the color. Nevertheless, in several cases, the confidence in the identification being correct is reinforced precisely because the color indices of the corresponding stars in the USNO catalogs are unrealistically blue. Many red variable stars with no charts can be reliably identified, because their positions closely coincide with those of objects in the infrared IRAS Point Source Catalog (Neugebauer et al. 1988), particularly if a high probability of variability was noted for the IRAS object; in most cases, the accuracy of the IRAS coordinates makes the identification with positional catalogs quite reliable. However, the "associations" ( positional coincidences, possible identifications) contained in the IRAS catalog are not always correct identifications, particularly in crowded star fields (in volume I of the GCVS, such cases are most common in Corona Australis).
Among the Harvard variable stars, there are many stars with the only finding chart published in the atlas by Tsesevich and Kazanasmas (1971). These valuable charts are generally based on Tsesevich's searches in the Harvard plate stacks in the 1950s. Unfortunately, mistakes are commonly found in the atlas (thus, among the many cases where two charts of the atlas show the same variable, there are occasions of different identifications suggested by the two charts, without any stipulation). Occasionally, the star marked in the chart is too far from the position published by its discoverer. In these cases, a check of the Harvard plates commonly allows a different, correct identification to be achieved. In addition, the hand-drawn charts of the atlas are difficult to use in crowded star fields. We tried to be cautious when using the charts by Tsesevich and Kazanasmas.

During the initial preparation of the GCVS version with improved coordinates, we made an attempt to automatically identify the GCVS with the Hubble Space Telescope Guide Star Catalog (GSC; Lasker et al. 1990), which revealed an unacceptably large number of incorrect or missed identifications. Therefore, we decided to check all identifications using finding charts. An independent solution of this problem at the SAI Department of Astrometry led to the compilation of a catalog by Gulyaev and Ashimbaeva (1997). Unfortunately, so far this catalog is accessible neither electronically nor in any other form. In addition, since the authors of the catalog compared the finding charts only with the GSC (a limiting magnitude no fainter than $15^{m}$ ), the catalog by Gulyaev and Ashimbaeva could not cover all GCVS stars with sufficient completeness and did not invoke deeper catalogs or measurements of actual sky images. By contrast, we additionally used identifications with the USNO A1.0/A2.0 catalogs (in favorable cases, their limiting magnitude reaches $23^{m}$ ) and measured coordinates for stars that were absent even in these catalogs.

To perform identifications using charts required tools for visualizing the astronomical catalogs. Our main tool was the SIMFOV code written by A.A. Volchkov (SAI). In its current form (A. Volchkov and O. Volchkov 2001), the code allows one to display the chart of a selected sky field on the required scale with objects from the catalogs in the specified list and then to view it, zooming in and out, and to retrieve the information contained in the corresponding catalog that refers to any image. This code also enables an automatic identification of user lists with catalogs by coordinates with (or without) allowance for the match between magnitudes. Viewing images, retrieving information, and comparing lists are possible for the coordinates that refer to any equinoxes. 
It has become possible to use the SIMFOV code for visualizing the USNO catalogs only recently, because it required much computer disk space. Previously, we used the special software for viewing the USNO A1.0/A2.0 catalogs directly from CD-ROMs that were kindly provided by J. Manek (Czech Republic).

The variable components of visual double and multiple systems required particular attention in preparing the catalog. In general, the components of sufficiently wide pairs are presented separately in positional catalogs and, thus, caused no difficulty. However, closer double and multiple stars in positional catalogs are often unresolved. In such cases, we used several approaches. For several variable components of double and multiple systems, we could measure the coordinates on Digitized Sky Survey images. In other cases, we applied a correction to the position of the double star's primary component based on the angular separation between the components and on the position angle at a certain epoch. Wherever possible, we also used data on the proper motions of each component or on the orbital motion in the system to reduce the coordinates to the epoch and equinox 2000.0.

\section{RESULTS}

The electronic version of Volume I is accessible at

ftp.sai.msu.su/pub/groups/cluster/gcvs/gcvs/voll/ or at

www.sai.msu.su/groups/cluster/gevs/gcvs/vol1/.

In its main table (voll.dat), the new electronic version of volume I of the GCVS contains information on 10558 objects (not counting the stars that were erroneously named for the second time or proved to be nonexistent) in Andromeda-Crux, which are mostly variable stars of our Galaxy discovered and named before 2001 ; i.e., it covers the variable stars that were included in the fourth edition of the GCVS and name lists nos. 67-76 (for the same constellations). For 10349 variable stars, our version provides new equatorial J2000.0 coordinates (voll_pos.dat), for 4587 stars with allowance for their proper motions; so far we have failed to determine accurate coordinates for 209 variable stars, because there are no finding charts and because the information for star identification is insufficient. The main table is supplemented with a list of remarks to volume I (rem.txt) published electronically for the first time and with a preliminary electronic version of bibliographic references (ref.txt) for volumes I-III of the 4 th GCVS edition. A detailed description of the files can be found in the readme.txt file of the electronic version.

\section{The File vol1.dat}

The structure of the main table (voll.dat) corresponds to that of the combined table of the GCVS 4th edition and the name lists (Kholopov et al. 1998; see also www.sai.msu.su/groups/cluster/gcvs/gcvs/iii/ iii.dat). The differences between these tables are as follows:

(1) Instead of B1950.0 coordinates, we provide new improved equatorial J2000.0 coordinates (right ascensions to within 0.1 and declinations to within $\left.1^{\prime \prime}\right)$. The coordinates that could not be improved were recalculated from old rough coordinates to the equinox J2000.0 with allowance for precession.

(2) The most recent name lists, up to no. 76 , were included.

(3) We included all the corrections and changes that were found during our work on the GCVS in other columns of the main table, in references, and in remarks. Note that for stars that had no published finding charts but that we identified with the GSC or with the US Naval Observatory (USNO) A1.0/A2.0 catalogs, we now give a symbol of the corresponding catalog (GSC, USNO) as a reference to the finding chart.

The main table is presented in the form traditional for the GCVS, i.e., in the order of constellations and variable-star names adopted in the GCVS. The table includes the following information: equatorial J2000.0 coordinates (right ascensions to within 0.1 and declinations to within $1^{\prime \prime}$ ), variability types, magnitudes at maximum and minimum light, photometric magnitude systems, periods of light variations, durations of brightness rise from minimum to maximum or eclipse durations, spectral types, and bibliography. For the stars from name lists nos. 67-76 published after the appearance of the GCVS 4th edition, we provide not all columns of the table but only coordinates, variability types, magnitudes, and bibliography; the missing data will be added to the 5 th edition of the GCVS.

\section{The File vol1_pos.dat}

The table vol1_pos.dat provides positional information based on our identifications with major astrometric catalogs, on published data, or on our new measurements (see below) for 10349 stars of the new version of volume I (including stars of the new name lists in the same constellations). The order of stars in this table is the same as that in the main table. It consists of the following columns:

(1) Ordinal star numbers in a system traditional for the electronic GCVS versions;

(2) GCVS star names; 
(3) Improved equatorial J2000.0 coordinates (right ascensions to within 0.01 and declinations to within $\left.0^{\prime \prime} .1\right)$;

(4) A flag indicating that the coordinates are actually rougher than the new accuracy standard adopted in the catalog, because we failed to determine better coordinates and to find them in source catalogs or in publications. In comparatively numerous cases, such coordinates were obtained by transforming B1950.0 coordinates from the IRAS Point Source Catalog (see below). The flag is a column (:) in the position that follows the coordinates;

(5) Proper motions (in arcseconds per year for both coordinates) to within 0 ".001 per year;

(6) Epochs of the given coordinates. No epoch is provided when using the coordinates from the literature if the epoch was not specified in the publication and could not be established;

(7) A flag indicating that the variable star's identification with the source catalog is uncertain (a question mark in the corresponding position);

(8) A brief designation of the source of astrometric data. In several cases, the catalog designation is followed by the symbol "+pm"; it implies that the position for a certain epoch was taken from the catalog and reduced to the epoch 2000.0 using information on the star's proper motion from a different source.

Below, we give a list of the major catalogs and other data sources used, roughly in the order of our preference in identifying variable stars (see also the description of the catalogs provided for A.A. Volchkov's visualization code we used at www.simfov.ru).

Hip-The Hipparcos Catalogue (ESA 1997).

Tyc2-The Tycho Catalogue (Høg et al. 2000). In isolated instances where the star is absent in the second Tycho catalogue but is present in the first catalogue (ESA 1997), the source is indicated as Tyc1.

PPM-The Positions and Proper Motions (Röser et al. 1991-1993).

NPM-The Lick Northern Proper Motion Program (Klemola et al. 1987).

AC-The Four-Million Star Catalog (see Gulyaev and Nesterov 1992).

ACT - The ACT Reference Catalog (Urban et al. 1997).

GSC-The Guide Star Catalog (Lasker et al. 1990). The symbol "ns" accompanying a GSC reference implies that the entry is marked as a nonstellar object in the cited catalog.

A2.0-A Catalog of Astrometric Standards (Monet et al. 1998). There are rare cases where the star could be found only in the previous version of the catalog; they are marked as A1.0.
IRAS-The Infrared Astronomical Satellite Catalogue of Point Sources (Neugebauer et al. 1988). We used it only when it was impossible to identify a star with positional catalogs or to find a star at optical wavelengths and independently measure its coordinates.

For some 200 stars $(1.8 \%$ of all the stars in the new version), we measured their coordinates using Digitized Sky Survey images, plates from various collections, or other images. In these cases, the source of coordinates is indicated as "GCVS authors." As a rule, we used A2.0 catalog stars as the reference stars in our measurements using the Digitized Sky Survey. The rectangular coordinates of stars were measured by an eye-pointing of the cursor at their photometric centers in commercially available image processing applications with a large magnification. The measurements were reduced by Turner's linear method. The accuracy of our measurements was typically about 0.5 both in right ascension and in declination. The coordinates taken from current astronomical periodicals are marked as "Literature."

We provide a portion (the first 20 stars) of the table vol1_pos.dat as a guide to its contents (Table 1).

The distribution of stars from the new GCVS version in the sources of their coordinates is shows in the figure; the sources IRAS, PPM, ACT, GCVS authors, and Literature were combined into a sector called "Others." The sector "Tyc2" also includes the rare cases of coordinates from the first version of the Tycho catalog (for the stars missing in the second version), and the sector "A2.0" includes the rare cases of coordinates from the first version of the corresponding US Naval Observatory catalog.

\section{The Files rem.txt and ref.txt}

The list of remarks to volume I (rem.txt) includes information that supplements the main table for 2070 stars. In contrast to the printed bilingual version of the 4th GCVS edition (Kholopov et al. 19851988), all remarks in the file are given only in English and generally correspond to the English text of the remarks in the printed edition. The remarks contain, in particular, information on variable stars in double stars, on period variability, on secondary minima of eclipsing variables, and on proximity to star clusters and nebulae in the sky. For unique variable stars, the remarks give detailed descriptions of the variability pattern.

The preliminary version of bibliographic references (ref.txt) for volumes I-III of the 4th edition contains 10200 references to the literature from the main table and remarks. All references in Cyrillic alphabet in the printed version were transliterated into the Latin alphabet in this file. 
Table 1. A portion of the electronic table vol1_pos.dat

\begin{tabular}{|c|c|c|c|c|}
\hline GCVS & J2000.0 & p.m. (as/yr) & Epoch & Source \\
\hline $010001 \mathrm{R}$ And & $002401.95+383437.3$ & $-0.016-0.035$ & 2000.0 & Hip \\
\hline $010002 \mathrm{~S}$ And & $004243.1+411605 .:$ & & 1885.67 & Literature \\
\hline $010003 \mathrm{~T}$ And & $002223.15+265945.8$ & $-0.007-0.003$ & 2000.0 & Тyc2 \\
\hline $010004 \mathrm{U}$ And & $011529.74+404307.7$ & $+0.014-0.017$ & 2000.0 & $\mathrm{AC}$ \\
\hline $010005 \mathrm{~V}$ And & $005006.28+353910.1$ & $-0.007-0.007$ & 2000.0 & NPM \\
\hline $010006 \mathrm{~W}$ And & $021732.96+441817.8$ & $-0.001-0.003$ & 2000.0 & Hip \\
\hline $010007 \times$ And & $001609.53+470045.3$ & $-0.000-0.000$ & 2000.0 & NPM \\
\hline $010008 \mathrm{Y}$ And & $013936.90+392034.7$ & & 1982.802 & GSC \\
\hline $010009 \mathrm{Z}$ And & $233339.95+484905.9$ & $-0.007-0.006$ & 2000.0 & Hip \\
\hline 010010 RR And & $005123.32+342236.8$ & $+0.011+0.002$ & 2000.0 & NPM \\
\hline 010011 RS And & $235521.75+483817.8$ & $+0.020-0.012$ & 2000.0 & Тyc2 \\
\hline 010012 RT And & $231110.10+530133.0$ & $-0.007-0.021$ & 2000.0 & Hip \\
\hline 010013 RU And & $013836.30+384013.5$ & $-0.004+0.007$ & 2000.0 & NPM \\
\hline 010014 RV And & $021102.57+485645.1$ & $+0.014-0.002$ & 2000.0 & Hip \\
\hline 010015 RW And & $004718.92+324108.8$ & $+0.011+0.017$ & 2000.0 & NPM \\
\hline 010016 RX And & $010435.52+411757.8$ & $+0.007-0.025$ & 2000.0 & NPM \\
\hline 010017 RY And & $232037.51+393713.9$ & $+0.002-0.011$ & 2000.0 & NPM \\
\hline 010018 RZ And & $230930.04+530239.8$ & $+0.004+0.001$ & 2000.0 & Hip \\
\hline 010019 SS And & $231130.07+525312.5$ & $-0.007-0.004$ & 2000.0 & Hip \\
\hline 010020 ST And & $233845.14+354621.2$ & $+0.002-0.004$ & 2000.0 & Hip \\
\hline
\end{tabular}

\section{REMARKS ABOUT SOME STARS OF THE ELECTRONIC VERSION}

Below, we give remarks pertaining to individual stars from volume I of the GCVS. We did not set the objective of listing in this paper all the complex cases we encountered during our work on the catalog. It seems to us that the examples collected in the remarks give an idea of the typical problems that the compilers of the catalog had to solve. The coordinates and identifications adopted for the stars described in the remarks are presented in Table 2.

NP And. The variable IV V122 discovered by Saha (1984) was erroneously labeled IV V108 in the discoverer's chart. The variable's coordinates presented in the same paper contradict the chart, pointing to a star of approximately the same brightness; its position relative to a brighter neighbor is symmetric with the variable. Our identification of NP And with GSC 2268.01354 corresponds to Saha's coordinates; a private communication from the author confirmed that the chart was erroneous.
AX Aps and LV Aps. The two stars are on the same chart with a small field of view published by the discoverer (Hoffmeister 1963). We failed to identify the chart with surrounding stars. A guess that the Mira variable LV Aps is identical to the infrared source IRAS 15491-7353 enabled us to identify the chart with a star field $20^{\prime}$ south of the published position.

CV Ara. Two charts from the atlas of Tsesevich and Kazanasmas (1971) were used to identify the variable with two different stars of the USNO A1.0 catalog: the first and second of them better match the variable's brightness and color (for an RR Lyrae variable), respectively. The color of the first star in the USNO A2.0 catalog is much bluer than in A1.0 and does not contradict the possible identification. The first star (GSC 9052.00158) is closer to the discoverer's coordinates (Swope 1936); Lopez (1998) identified the variable with this star. We adopted the same identification, although the uncertainty remains.

MX Ara. In the GCVS, this star is assumed to be 
Table 2. Examples of the coordinates and identifications for problem stars

\begin{tabular}{l|c|c|c|c|c|l|l}
\hline \multicolumn{1}{c|}{ Star } & $\alpha_{2000.0}$ & $\delta_{2000.0}$ & Epoch & $\mu_{\alpha}$ & $\mu_{\delta}$ & Source & \multicolumn{1}{|c}{ Identifications } \\
\hline NP And & $00^{h} 10^{m} 39.84$ & $+34^{\circ} 06^{\prime} 55^{\prime \prime} .7$ & 1982.800 & - & - & GSC & GSC 2268.01354 \\
AX Aps & 155509.19 & -740223.4 & 1976.236 & - & - & A2.0 & \\
LV Aps & 155501.76 & -740228.8 & 1991.25 & - & - & Tyc2 & Tyc2 9272 1590 1; IRAS 15491-7353 \\
CV Ara & 171317.13 & -611228.4 & 1976.632 & - & - & GSC & GSC 9052.00158? \\
MX Ara & 172209.78 & -594021.4 & 1976.633 & - & - & A2.0 & \\
NZ Ara & 173750.04 & -593731.0 & 1975.450 & - & - & A2.0 & Uncertain identification \\
RR Cnc & 081103.15 & +230854.6 & 2000.0 & $-0^{\prime \prime} 008$ & $-0^{\prime \prime} 010$ & Tyc2 & Tyc2 1927 1971 1; GSC 1927.01961; \\
V CMa & 064340.70 & -314656.5 & 2000.0 & +0.014 & -0.006 & Tyc2 & CoD-31 3607? Tyc2 7087 114 1; \\
EV CMa & 071934.07 & -194808.3 & 1980.211 & - & - & A2.0 & \\
V535 Cen & 143727.39 & -370301.8 & 2000.0 & -0.022 & -0.027 & AC & GSC 7308.01701 \\
AV Cha & 123709.08 & -771817.7 & 1976.250 & - & - & A2.0 & IRAS 12338-7701 \\
SU Col & 050747.05 & -335154.5 & 2000.0 & +0.004 & -0.011 & Hip & HIP 23868; CoD-34 2083; \\
V359 CrA & 181603.27 & -383905.5 & 2000.0 & -0.011 & -0.006 & Tyc2 & V530 CrA; Tyc2 7900 2773 1; \\
\end{tabular}

identical both to the variable HV 7632 (Shapley and Boyd 1937) and to the variable S 6161 (Hoffmeister 1963). At close coordinates, these stars had the same variability type, and Hoffmeister's chart did not contradict the description given by Shapley and Boyd (the northern preceding star of the pair varies). We adopted Hoffmeister's identification. It should be noted, however, that Tsesevich and Kazanasmas (1971) suggested another star, which is not in conflict with the description either.

NZ Ara. The chart of Tsesevich and Kazanasmas (1971) shows severe distortions. Our identification takes into account the coordinates, brightness, and the chart but is uncertain.

RR Cnc. The star is confidently identified with GSC 1927.01961. However, in the Tycho (ESA 1997) and Tycho-2 (Høg et al. 2000) catalogs, contrary to the declared preservation of the designations for GSC stars in the latter, the star number is Tyc 192719711.

V CMa. There is no finding chart. The first and second editions of the well-known reference book "Geschichte und Literatur..." (GuL; Müller and
Hartwig 1918; Prager 1934) give conflicting and erroneous (in both cases) descriptions of the star's duplicity, which completely confuse the identification. The star was found on Harvard Observatory plates using the discoverer's (A. Cannon) notebooks, and its variability was confirmed.

EV CMa. The discoverer's chart (Van Hoof 1961) cannot be identified with the sky at the specified position. There is a blot at the place of the cited paper where the right ascension is given, suggesting a misprint. The star was found 9 min of time (approximately two degrees of arc) to the east from the previously adopted coordinates.

WY Car. An example of a problem case where the coordinates cannot yet be improved. (We did not include WY Car in the table.) The first edition of GuL reports that the star is missing from the CPD catalog, whereas the second edition of GuL identifies it with $\mathrm{CoD}-57^{\circ} 3577$. This $\mathrm{CoD}$ star is almost certain to be identical to CPD-57 3969 and HD 303467, an F0-type star. This identification of the faint longperiod (semiregular or Mira-type) variable WY Car is acceptable neither in brightness nor in spectral type and color. The possible candidates in a wide field have not yet been confirmed. 


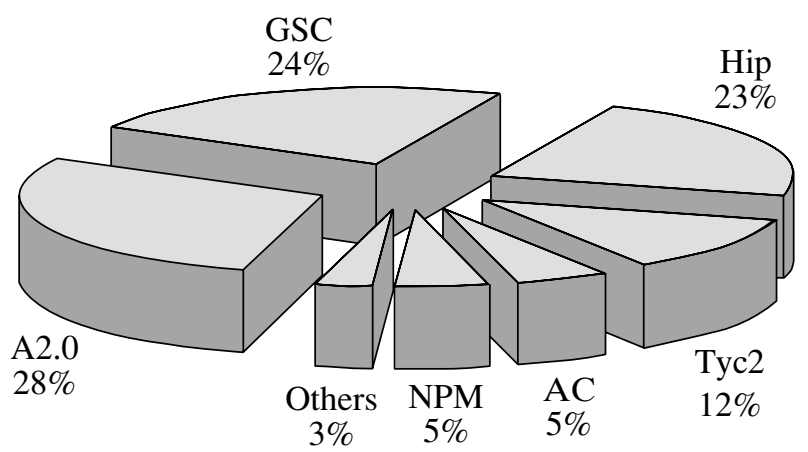

The sector diagram showing the distribution of stars from the new GCVS version in the sources of accurate coordinates.

V535 Cen. The choice between the two conflicting charts from the atlas of Tsesevich and Kazanasmas (1971) required a check in the Harvard Observatory plate stacks.

AV Cha. The chart from Hoffmeister (1963) allows the star to be identified with NSV 05771 (HV $8452=$ 188.1933; Luyten 1933). Hoffmeister also noticed that the variable he discovered was close to Luyten's star but did not identify them because of the large error in his coordinates of AV Cha ( $\sim 3^{\prime}$ in declination).

SU Col. The GCVS gave an erroneous type for this variable, CWA, with a period of $21 d 55$, according to Avery and Sievers (1968). Actually, the star is an RR Lyrae variable with a period of 0.487 (Gessner 1985). The chart in the cited paper by Avery and Sievers is also erroneous. However, the identification causes no difficulty, because accurate coordinates and the light curve can be found in the Hipparcos catalogue (ESA 1997).

GT Com. Schmeer (1999) found that this flare star could actually be the minor planet (679) Pax. We confirmed the object's motion among stars on the Maria Mitchell Observatory (USA) plates that were used by D. Hofleit to discover the variable object (Nygard 1973). The object was excluded from the GCVS as a nonexistent star; we did not include it in the table.

V359 CrA and V530 CrA. The coordinates of these stars published by their discoverers (Swope 1943; Kooreman 1966) differed by 1 min of time in right ascension and by $0.7 \mathrm{~min}$ of arc in declination. However, the chart for V359 CrA (Tsesevich and Kazanasmas 1971) pointed to the same star as Kooreman's chart for V530 CrA, with Kooreman's coordinates being approximately correct. Our confidence in the identification of the two variables stems from the fact that the epoch of minimum published by Swope for the first eclipsing variable does not contradict Kooreman's elements for the second one.
The stars were declared identical in the new GCVS version.

\section{ACKNOWLEDGMENTS}

We are grateful to A.A. Volchkov and J. Manek for providing us with the necessary software. We wish to thank S.V. Antipin for assistance, L.N. Berdnikov for providing unpublished finding charts for several southern Cepheids, C. Lopez (Argentina), N.T. Ashimbaeva, and G.V. Romanova for making their results available to us prior to their publication. We also wish to thank the researchers, primarily G. Williams (USA), who helped us to identify the "lost" variable stars using plates of other observatories. This study was supported in part by the Russian Foundation for Basic Research (project no. 99-0216333), the Program of Support for Leading Scientific Schools of Russia (project no. 00-15-96627), the Federal Program "Astronomy," and the American Association of Variable Star Observers. We used the Digitized Sky Survey images provided by the Hubble Space Telescope Science Institute under support from grant NAG W-2166 of the USA Government.

\section{REFERENCES}

1. R. Avery and J. Sievers, Veröff. Sternw. Bamberg. 7 (76), (1968).

2. H. Gessner, Inf. Bull. Var. Stars, No. 2795 (1985).

3. A. P. Gulyaev and N. T. Ashimbaeva, Astron. Zh. 74, 249 (1997) [Astron. Rep. 41, 215 (1997)].

4. C. Hoffmeister, Veröff. Sternwarte Sonneberg 6, 1 (1963).

5. E. Høg, C. Fabricius, V. V. Makarov, et al., The Tycho-2 Catalogue, CD-ROM (Copenhagen, 2000).

6. P. N. Kholopov, N. N. Samus', M. S. Frolov, V. P. Goranskilı, N. A. Gorynya, E. A. Karitskaya, E. V. Kazarovets, N. N. Kireeva, N. P. Kukarkina, N. E. Kurochkin, G. I. Medvedeva, N. B. Perova, A. S. Rastorguev, and S. Yu. Shugarov, General Catalogue of Variable Stars (Nauka, Moscow, 19851988), Vols. I-III.

7. P. N. Kholopov, N. N. Samus, N. M. Artiukhina, O. V. Durlevich, M. S. Frolov, V. P. Goranskij, N. A. Gorynya, E. A. Karitskaya, E. V. Kazarovets, N. N. Kireeva, N. P. Kukarkina, N. E. Kurochkin, N. A. Lipunova, G. I. Medvedeva, E. N. Pastukhova, N. B. Perova, A. S. Rastorguev, S. Yu. Shugarov, and T. M. Tsvetkova, Centre de Données Astronomiques de Strasbourg, II/214A (1998).

8. A. R. Klemola, R. B. Hanson, and B. F. Jones, Centre de Données Astronomiques de Strasbourg, I/199 (1987).

9. C. J. Kooreman, Ann. Sterrew. Leiden. 22, 159 (1966).

10. B. M. Lasker, C. R. Sturch, B. J. McLean, et al., Astron. J. 99, 2019 (1990). 
11. C. Lopez, private communication (1998).

12. W. J. Luyten, Astron. Nachr. 249, 395 (1933).

13. D. Monet, A. Bird, B. Canzian, et al., USNO-A V2.0. A Catalog of Astrometric Standards (US Naval Observatory, Washington, 1998), 11 CD ROMs.

14. G. Müller and E. Hartwig, Geschichte und Literatur... (Poeschel \& Trepte, Leipzig, 1918), vol. 1.

15. G. Neugebauer, R. J. van Duinen, H. J. Habing, et al., IRAS Point Source Catalog (NASA, Washington, 1988).

16. S. Nygard, J. Am. Assoc. Var. Stars Obs. 2, 60 (1973).

17. On the Four-Million Star Catalog, Ed. by A. P. Gulyaev and V. V. Nesterov (Mosk. Gos. Univ., Moscow, 1992).

18. R. Prager, Geschichte und Literatur des Lichtwechsels der Veränderlichen Sterne, 2. Ausgabe (F. Dümmler, Berlin, 1934), vol. 1.

19. S. Röser, U. Bastian, L. I. Yagudin, and V. V. Nesterov, PPM Star Catalogue. Positions and Proper Motions (Astronomisches Rechen-Institut, Heidelberg, 1991-1993).
20. A. Saha, Astrophys. J. 283, 580 (1984).

21. P. Schmeer, private communication (1999).

22. H. Shapley and C. D. Boyd, Ann. Harvard Obs. 105, 243 (1937).

23. H. H. Swope, Ann. Harvard Obs. 90, 207 (1936).

24. H. H. Swope, Ann. Harvard Obs. 109, 41 (1943).

25. The Hipparcos and Tycho Catalogues (European Space Agency, 1997), ESA SP-1200.

26. V. P. Tsesevich and M. S. Kazanasmas, Atlas of Finding Charts for Variable Stars (Nauka, Moscow, 1971).

27. S. E. Urban, T. E. Corbin, and G. L. Wyckoff, The ACT Reference Catalog (US Naval Obs., Washington, 1997).

28. A. van Hoof, Publ. Louvain Obs., No. 126 (1961).

29. A. A. Volchkov and O. A. Volchkov, http://www.simfov.ru, 2001.

Translated by N. Samus' 